\title{
Induction of Modular Classification Rules: Using Jmax-pruning
}

\author{
Frederic Stahl and Max Bramer
}

\begin{abstract}
The Prism family of algorithms induces modular classification rules which, in contrast to decision tree induction algorithms, do not necessarily fit together into a decision tree structure. Classifiers induced by Prism algorithms achieve a comparable accuracy compared with decision trees and in some cases even outperform decision trees. Both kinds of algorithms tend to overfit on large and noisy datasets and this has led to the development of pruning methods. Pruning methods use various metrics to truncate decision trees or to eliminate whole rules or single rule terms from a Prism rule set. For decision trees many pre-pruning and postpruning methods exist, however for Prism algorithms only one pre-pruning method has been developed, $J$-pruning. Recent work with Prism algorithms examined $J$ pruning in the context of very large datasets and found that the current method does not use its full potential. This paper revisits the J-pruning method for the Prism family of algorithms and develops a new pruning method Jmax-pruning, discusses it in theoretical terms and evaluates it empirically.
\end{abstract}

\section{Introduction}

Classification rule induction from large training samples has a growing commercial importance and can be traced back to the 1960s [7]. Two general approaches to classification rule induction exist the 'separate and conquer' and the 'divide and conquer' approaches [14]. 'Divide and conquer' is better known as Top Down Induction of Decision Trees (TDIDT) [10] as it induces classification rules in the intermediate representation of a decision tree. The 'separate and conquer' approach can be traced back to the AQ learning system in the 1960s [9], however most research concentrates on the TDIDT approach.

Frederic Stahl, Max Bramer

University of Portsmouth, School of Computing, Buckingham Building, Lion Terrace, PO1 3HE Portsmouth, UK e-mail: \{Frederic.Stahl; Max.Bramer\} @ port.ac.uk 
An important development of the 'separate and conquer' approach is the Prism family of algorithms [5, 2, 3]. Prism induces rules that are modular and that do not necessarily fit into a decision tree. Prism achieves a comparable classification accuracy compared with TDIDT and in some cases even outperforms TDIDT [2], especially if the training data is noisy. Recent research on the Prism family of algorithms comprises a framework that allows the parallelisation of any algorithm of the Prism family in order to make Prism algorithms scale better to large training data. The framework is called Parallel Modular Classification Rule Inducer (PMCRI) [13].

Like any classification rule induction algorithm Prism suffers from overfitting rules to the training data. Overfitting can result in a low predictive accuracy on previously unseen data instances (the test set) and a high number of induced rules and rule terms. There exist a variety of pruning methods for decision trees [6] that aim to reduce the unwanted overfitting, however there is only one published method of pruning rules of the Prism family, J-pruning [3]. J-pruning uses the $J$-measure, an information theoretic means to quantify the information content of a rule. J-pruning pre-prunes the rules during their induction. J-pruning has been integrated in PMCRI and not only improves the predictive accuracy but also lowers the number of rules and rule terms induced and thus also improves the computational efficiency of Prism algorithms [12].

This paper revisits the J-measure and J-pruning, develops Jmax-pruning, a variation of J-pruning and evaluates them empirically. Section 2 outlines the Prism Family of algorithms and compares them to TDIDT. Section 3 outlines Jmax-pruning followed by an empirical evaluation in Section 4. Ongoing work is discussed in Section 5 which comprises a new variation of the Prism approach and Jmax-pruning for TDIDT. Some concluding remarks can be found in Section 6.

\section{The Prism Family of Algorithms}

As mentioned in Section 1, rule representation differs between the 'divide and conquer' and 'separate and conquer' approaches in that the rule sets generated by the 'divide and conquer' approach are in the form of decision trees whereas rules generated by the 'separate and conquer' approach are modular, do not necessarily fit into a decision tree and normally do not. The rule representation of decision trees is the main drawback of the 'divide and conquer' approach, for example rules such as:

$$
\begin{aligned}
& \text { IF } a=1 \text { AND } b=1 \text { THEN class }=1 \\
& \text { IF } c=1 \text { AND } d=1 \text { THEN class }=0
\end{aligned}
$$

cannot be represented in a tree structure as they have no attribute in common. Forcing these rules into a tree will require the introduction of additional rule terms that are logically redundant, and thus result in unnecessarily large and confusing trees [5]. This is also known as the replicated subtree problem [14].

'Separate and conquer' algorithms induce directly sets of 'modular' rules like those above avoiding unnecessarily redundant rule terms that are induced just for 
the representation in a tree structure. The basic 'separate and conquer' approach can be described as follows:

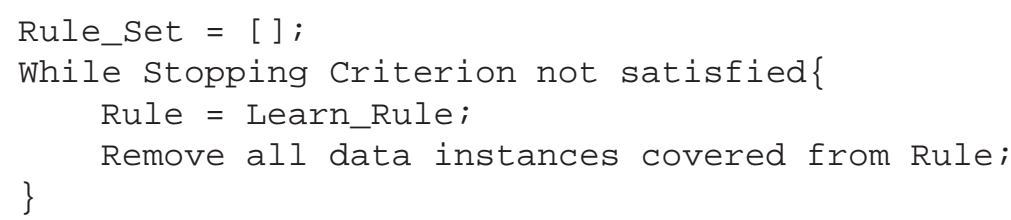

The Learn_Rule procedure generates the best rule for the current subset of the training data where best is defined by a particular heuristic that may vary from algorithm to algorithm. The stopping criterion is also dependent on the algorithm used. After inducing a rule, the rule is added to the rule set and all instances that are covered by the rule are deleted and a new rule is induced on the remaining training instances.

In Prism each rule is generated for a particular Target Class (TC). The heuristic Prism uses in order to specialise a rule is the probability with which the rule covers the TC in the current subset of the training data. The stopping criterion is fulfilled as soon as there are no training instances left that are associated with the TC.

Cendrowska's original Prism algorithm selects one class as the TC at the beginning and induces all rules for that class. It then selects the next class as TC and resets the whole training data to its original size and induces all rules for the next TC. This is repeated until all classes have been selected as TC. Variations exist such as PrismTC [4] and PrismTCS (Target Class Smallest first) [3]. Both select the TC anew after each rule induced. PrismTC always uses the majority class and PrismTCS uses the minority class. Both variations introduce an order in which the rules are induced, where there is none in the basic Prism approach. However the predictive accuracy of PrismTC cannot compete with that of Prism and PrismTCS (personal communication). PrismTCS does not reset the dataset to its original size and thus is faster than Prism, which produces a high classification accuracy and also sets an order in which the rules should be applied to the test set.

The basic PrismTCS algorithm is outlined below where $A_{x}$ is a possible attribute value pair and $D$ is the training dataset:

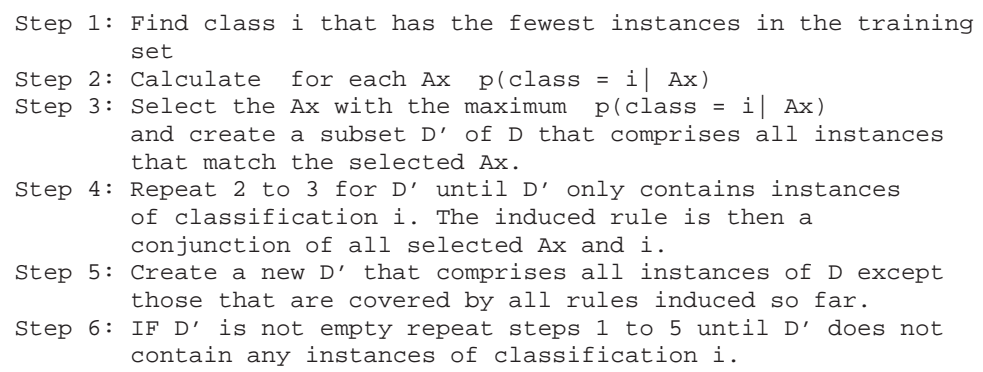

We will concentrate here on the more popular PrismTCS approach but all techniques and methods outlined here can be applied to any member of the Prism family. 


\subsection{Dealing with Clashes}

A clash set is a set of instances in a subset of the training set that are assigned to different classes but cannot be separated further. For example this is inevitable if two or more instances are identical except for their classification. Cendrowska's original Prism algorithm does not take into account that there may be clashes in the training data. However the Inducer software implementations of the Prism algorithms do take clashes into account $[2,4]$. What happens in the case of a clash in Inducer is that all instances are treated as if they belong to the TC. [2] mentions that the best approach is to check if the TC is also the majority class. If it is then the rule currently being induced is taken otherwise the rule is discarded. If a clash is encountered and the majority class is not the TC then the rule is discarded and all instances in the clash set that match the TC are deleted. The reason for manipulating the clash set this way is that if the rule were discarded and the clash set kept then the same rule would be induced all over again and the same clash set would be encountered again.

\subsection{Dealing with Continuous Attributes}

Continuous attributes are not handled by Cendrowska's original Prism algorithm. One way to deal with continuous attributes is discretisation of the attribute values prior to the algorithm's application, for example applying ChiMerge [8] before the application of a Prism algorithm. Bramer's Inducer software [4] provides implementations of Prism algorithms that deal with continuous attributes, these are also used in all Prism implementations used in this work. Dealing with continuous attributes can be integrated in step two in the pseudo code above before the calculation of $p\left(\right.$ class $\left.=i \mid A_{x}\right)$. If $A_{x}$ is continuous then the training data is sorted for $A_{x}$. For example let $A_{x}$ comprise the following values after sorting, -3, -4.2, 3.5, 5.5 and 10, then the data is scanned for these attribute values in either ascending or descending order. For each attribute value, for example 5.5, two tests $p\left(\right.$ class $\left.=i \mid A_{x}<5.5\right)$ and $p\left(\right.$ class $\left.=i \mid A_{x} \geq 5.5\right)$ are conducted. The one with the largest conditional probability for all the values of the attribute is kept and compared with those conditional probabilities from the remaining attributes.

\subsection{J-pruning}

As mentioned in the introduction, classifiers are generally pruned to prevent them from overfitting. Pruning methods can be divided into two categories, pre-pruning and post-pruning. Post-pruning is applied to the classifier after it has been induced whereas pre-pruning is applied during the rule induction process. For Prism algorithms only one pruning method has been developed so far, J-pruning [3], a prepruning method based on the J-measure [11], a measure for the information content 
of a rule. J-pruning can also be applied to decision tree induction algorithms and has shown good results on both kinds of algorithms [3]. As also mentioned in the introduction, J-pruning has found recent popularity in [12], as it reduces the number of rules and rule terms induced considerably and thus increases the computational efficiency.

According to [11] the theoretical average information content of a rule of the form $I F Y=y$ THEN $X=x$ can be measured in bits and is denoted by $J(X, Y=y)$.

$$
J(X ; Y=y)=p(y) \cdot j(X ; Y=y)
$$

$J(X ; Y=y)$ is essentially a product of $p(y)$, the probability with which the left hand side of the rule will occur, and $j(X ; Y=y)$ which is called the j-measure (with a lower case $j$ ) and measures the goodness-of-fit of a rule. The $j$-measure, also called the cross-entropy, is defined as:

$$
j(X ; Y=y)=p(x \mid y) \cdot \log _{2}\left(\frac{p(x \mid y)}{p(x)}\right)+(1-p(x \mid y)) \cdot \log _{2}\left(\frac{(1-p(x \mid y))}{(1-p(x))}\right)
$$

For a more detailed description of the J-measure Smyth's paper [11] is recommended. Bramer's essential interpretation of the J-measure is that if a rule has a high J-value then it also is likely to have a high predictive accuracy [3]. Hence the $\mathrm{J}$-value is used as an indicator of whether appending further rule terms is likely to improve a rule's predictive accuracy or lower it due to overfitting. The J-value of the rule may go up or down when appending rule terms, also it may go down and up again. However it is possible to calculate the maximum J-value that the rule with its current terms might maximally achieve if additional terms were added. This upper bound cannot of course be exceeded but its value is not necessarily achievable.

Bramer's basic J-pruning is applied to Prism by calculating the J-value of the rule before the induction of a new rule term and the J-value that the rule would have after a newly induced rule term is appended. If the J-value goes up then the rule term is appended. In the case where the J-value goes down, the rule term is not appended and a test is applied to determine whether the majority class of the instances that are covered by the rule is also the TC. If the majority class is the TC then the rule is truncated and kept and all instances in the current subset of the training set are treated as if all instances belong the TC. If the majority class is not the TC, then the rule is discarded and the clash resolution described in Section 2.1 is invoked.

\section{Variation of J-pruning}

In general there is very little work on pruning methods for the Prism family of algorithms. Bramer's J-pruning in the Inducer software seems to be the only pruning facility developed for Prism algorithms. This section critiques the initial J-pruning facility and outlines Jmax-pruning, a variation that makes further use of the J-measure. 


\subsection{Critique of J-pruning}

Even though J-pruning described in Section 2.3 achieves good results regarding the overfitting of Prism, it does not seem to exploit the J-measure to its full potential. The reason is that even if the new rule term decreases the J-value, it is possible that the J-value increases again when adding further rule terms [12]. If the rule is truncated as soon as the J-value is decreased it may result in the opposite of overfitting, an over generalised rule with a lower predictive accuracy. The relatively good results for J-pruning achieved in [3] could be explained by the assumption that it does not happen very often that the J-value decreases and then increases again. However, how often this happens will be examined empirically in Section 4.

\subsection{Jmax-pruning}

According to [11], an upper bound for the J-measure for a rule can be calculated by:

$$
J_{\max }=p(y) \cdot \max \left\{p(x \mid y) \cdot \log _{2}\left(\frac{1}{p(x)}\right),(1-p(x \mid y)) \cdot \log _{2}(1 \mid 1-p(x))\right\}
$$

If the actual J-value of the rule currently being generated term by term matches the maximum possible J-value $\left(J_{\max }\right)$ it is an absolute signal to stop the induction of further rule terms.

A concrete example is used to show how the J-values of a rule can develop. The example used a dataset extracted from the UCI repository, the soybean dataset [1]. Here we induce rules using our own implementation of PrismTCS without any $J$ pruning. The original dataset has been converted to a training set and a test set where the training set comprises $80 \%$ of the data instances. The 39 th rule induced is:

$$
\begin{gathered}
\text { IF }(\text { temp }=\text { norm }) \text { AND }(\text { same-lst-sev-yrs }=\text { whole-field }) \text { AND }(\text { crop-hist }= \\
\text { same-lst-two-yrs }) \text { THEN CLASS }=\text { frog-eye-leaf-spot }
\end{gathered}
$$

This is a perfectly reasonable rule with a J-value of 0.00578 . However looking at the development of the J-values after each rule term appended draws a different picture:

\section{$\underline{\text { First Term }}$}

$$
\begin{gathered}
\text { IF }(\text { temp }=\text { norm }) \text { THEN CLASS }=\text { frog-eye-leaf-spot } \\
\left(\mathrm{J} \text {-value }=0.00113, J_{\max }=0.02315\right)
\end{gathered}
$$

Here the rule has J-value of 0.00113 after the first rule term has been appended. The J-value for the complete rule (0.00578) is larger than the current J-value, which is to be expected as the rule is not fully specialised yet on the TC. 


\section{$\underline{\text { Second Term }}$}

$$
\begin{gathered}
\text { IF }(\text { temp }=\text { norm }) \text { AND }\left(\begin{array}{c}
\text { same-lst-sev-yrs }=\text { whole-field }) \text { THEN CLASS }= \\
\text { frog-eye-leaf-spot }
\end{array}\right. \\
\left(\mathrm{J} \text {-value }=0.00032, J_{\max }=0.01157\right)
\end{gathered}
$$

Now the J-value is decreased to 0.00032 and $J_{\max }$ to 0.01157 . Here $J$-pruning as described in Section 2.3 would stop inducing further rule terms, the finished rule would be

$$
\text { IF }(\text { temp }=\text { norm }) \text { THEN CLASS }=\text { frog-eye-leaf-spot }
$$

with a J-value of 0.00113 . However looking at the value of $J_{\max }$, after the second rule term has been appended, it can be seen that it is still higher than the previous $\mathrm{J}$-value for appending the first rule term. Thus it is still possible that the J-value may increase again above the so far highest J-value of 0.00113 . Inducing the next rule term leads to:

\section{Third Term}

$$
\begin{gathered}
\text { IF }(\text { temp }=\text { norm }) A N D(\text { same-lst-sev-yrs }=\text { whole-field }) \text { AND }(\text { crop-hist }= \\
\text { same-lst-two-yrs }) \text { THEN CLASS }=\text { frog-eye-leaf-spot } \\
\left(\mathrm{J} \text {-value }=0.00578, J_{\max }=0.00578\right)
\end{gathered}
$$

In this case the rule was finished after the appending of the third rule term as it only covered examples of the TC. However, the interesting part is that the Jvalue increased again by appending the third rule term and is in fact the highest J-value obtained. Using Bramer's original method would have truncated the rule too early leading to an overall average information content of 0.00113 instead of 0.00578 . The J-value and the Jmax value are rounded to five digits after the decimal point and appear identical but are actually slightly different. Looking at more digits the values are in fact for the J-value 0.005787394940853119 and for the $J_{\max }$ 0.005787395266794598 . In this case no further rule terms can be added to the lefthand side of the rule as the current subset of the training set only contains instances of the TC, but if this were not the case it would still not be worthwhile to add additional terms as the J-value is so close to Jmax.

Overall this observation strongly suggests that pruning the rule as soon as the Jvalue decreases does not fully exploit the J-measure's potential. This work suggests that $J$-pruning could be improved by inducing the maximum number of possible rule terms until the current subset cannot be broken down further or the actual Jvalue is equal to or within a few percent of Jmax. As a rule is generated all the terms are labelled with the actual J-value of the rule after appending this particular rule term. The partial rule for which the largest rule J-value was calculated would then 
be identified and all rule terms appended afterwards truncated, with clash handling as described in Section 2.1 invoked for the truncated rule [12]. We call this new pre-pruning method Jmax-pruning.

\section{Evaluation of Jmax-pruning}

The datasets used have been retrieved from the UCI repository [1]. Each dataset is divided into a test set holding $20 \%$ of the instances and a training set holding the remaining $80 \%$ of the instances.

Table 1 shows the number of rules induced per training set and the achieved accuracy on the test set using PrismTCS with J-pruning as described in Section 2.3 and Jmax-pruning as proposed in Section 3.2.

Table 1 Comparison of J-pruning and Jmax-pruning on PrismTCS.

\begin{tabular}{|c|c|c|c|c|c|}
\hline \multirow[t]{2}{*}{ Dataset } & Number of Rules & Accuracy (\%) & Number of Rules & Accuracy (\%) & \multirow[t]{2}{*}{ J-value recovers } \\
\hline & J-Prun & & J-max Pr & runing & \\
\hline monk1 & 4 & 79 & 12 & 86 & 4 \\
\hline monk3 & 3 & 98 & 3 & 98 & 0 \\
\hline vote & 3 & 94 & 3 & 94 & 0 \\
\hline genetics & 8 & 70 & 8 & 70 & 0 \\
\hline $\begin{array}{l}\text { contact } \\
\text { lenses }\end{array}$ & 4 & 95 & 4 & 95 & \\
\hline $\begin{array}{l}\text { breast } \\
\text { cancer }\end{array}$ & 24 & 96 & 24 & 96 & 0 \\
\hline soybean & 39 & 88 & 43 & 89 & 4 \\
\hline $\begin{array}{l}\text { australian } \\
\text { credit }\end{array}$ & 20 & 89 & 20 & 89 & 0 \\
\hline diabetes & 29 & 75 & 31 & 76 & 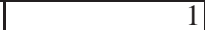 \\
\hline $\operatorname{crx}$ & 18 & 83 & 18 & 83 & 0 \\
\hline segmentation & 83 & 79 & 86 & 82 & 2 \\
\hline ecoli & 23 & 78 & 26 & 78 & 3 \\
\hline $\begin{array}{l}\text { Balance } \\
\text { Scale }\end{array}$ & 10 & 72 & 36 & 74 & 21 \\
\hline $\begin{array}{l}\text { Car } \\
\text { Evaluation }\end{array}$ & 4 & 76 & 4 & 76 & 1 \\
\hline $\begin{array}{l}\text { Contraceptive } \\
\text { Method } \\
\text { Choice }\end{array}$ & 19 & 44 & 28 & 45 & 8 \\
\hline $\begin{array}{l}\text { Optical } \\
\text { Recognition } \\
\text { of } \\
\text { handwritten } \\
\text { Digits }\end{array}$ & 456 & 57 & 467 & 58 & 6 \\
\hline
\end{tabular}

What is also listed in Table 1 as 'J-value recovers', is the number of times the $\mathrm{J}$-value decreased and eventually increased again when first fully expanding the rule 
and then pruning it using Jmax-pruning. Using the original J-pruning as described in Section 2.3 would not detect these J-value recoveries and lead to a rule with a lower J-value and thus lower information content than it could possibly achieve.

What can be seen is that in all cases Jmax-pruning performs either better than or produces the same accuracy as J-pruning. In fact seven times Jmax-pruning produced a better result than $J$-pruning and nine times it produced the same accuracy as $J$-pruning. Taking a closer look in the rule sets that have been produced in the nine cases for which the accuracies for both pruning methods are the same revealed that identical rule sets were produced in seven out of these nine cases. The two exceptions are the 'Car Evolution' and 'ecoli' datasets, however in these two exceptions the classification accuracy was the same using Jmax-pruning or J-pruning. In the cases where there are identical classifiers there were no J-value recoveries present. In Section 3.1 we stated that the good performance of J-pruning [3], despite its tendency to generalisation, can be explained by the fact that there are not many J-value recoveries in the datasets and thus the tendency to over generalisation is low. Looking into the last column of table 1 we can see the number of J-value recoveries. In seven cases there are none, thus there is no potential for over generalisation by using $J$-pruning and for the remaining datasets there is only a very small number of J-value recoveries with the exception of the 'Balanced Scale' dataset for which a $2 \%$ higher accuracy has been retrieved by using Jmax-pruning compared with J-pruning.

Loosely speaking, if there are no J-value recoveries present, then Prism algorithms with Jmax-pruning will produce identical classifiers to Prism algorithms with $J$-pruning. However, if there are J-value recoveries, it is likely that Prism algorithms with Jmax-pruning will produce classifiers that achieve a better accuracy than Prism algorithms with J-pruning.

What can also be read from Table 1 is the number of rules induced. In all cases in which both pruning methods produced the same accuracy, the classifiers and thus the number of rules were identical. However in the cases where the J-value recovered, then the number of rules induced with Jmax-pruning was larger than the number of rules induced with $J$-pruning. This can be explained by the fact that in the case of a J-value recovery the rule gets specialised further than with normal $J$-pruning by adding more rule terms while still avoiding overfitting. Adding more rule terms results in the rule covering fewer training instances from the current subset. This in turn results in that before the next iteration for the next rule less instances are deleted from the training set, which potentially generates more rules, assuming that the larger the number of training instances the more rules are generated. 


\section{Ongoing Work}

\subsection{J-PrismTCS}

Annother possible variation of PrismTCS that is currently being implemented is a version that is solely based on the J-measure. Rule terms would be induced by generating all possible categorical and continuous rule terms and selecting the one that results in the highest J-value for the current rule instead of selecting the one with the largest conditional probability. Again the same stopping criterion as for standard PrismTCS could be used, which is that all instances of the current subset of the training set belong to the same class. We call this variation of PrismTCS, J-PrismTCS.

\subsection{Jmax-Pruning for TDIDT}

J-pruning has also been integrated into the TDIDT approach as a pre-pruning facility and achieved a higher classification accuracy than TDIDT without J-pruning [3]. Encouraged by the good results outlined in Section 4 which were achieved with Jmax-pruning in PrismTCS, we are currently developing a version of Jmax-pruning for TDIDT algorithms. The following pseudo code describes the basic TDIDT algorithm.

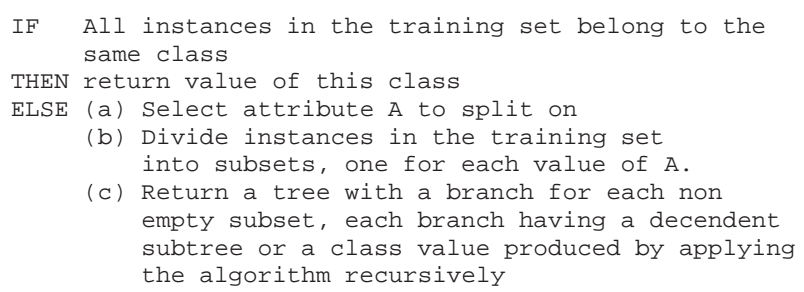

The basic approach of $J$-pruning in TDIDT is to prune a branch in the tree as soon as a node is generated at which the J-value is less than at its parent node [3]. However performing the J-pruning is more complicated than for Prism algorithms as illustrated in the example below.

Figure 1 illustrates a possible tree which is used to explain J-pruning and Jmaxpruning for TDIDT. The nodes labelled with '?' are placeholders for possible subtrees. Now assuming that a depth first approach is used and the current node being expanded is node 'D'. In this case $J$-pruning would take the incomplete rule

$$
\text { (1) } \operatorname{IF}(A=0) \text { AND }(B=0) \text { AND }(C=0) \ldots
$$

the complete rule

(2) IF $(A=0)$ AND $(B=0)$ AND $(C=0)$ AND $(D=0)$ THEN class $=1$ 


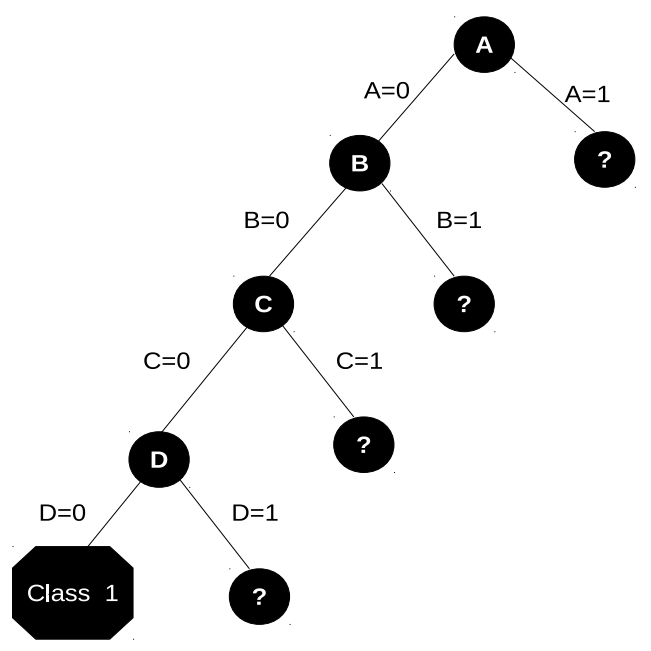

Fig. 1 Example of a decision tree for J-pruning.

and the possible incomplete rule

$$
\text { (3) IF }(A=0) \text { AND }(B=0) \text { AND }(C=1) \text { AND }(D=1) \ldots
$$

into account. Rule (2) is completed as all instances correspond to the same classification which is (class $=1$ ). However instances covered by incomplete rule (3) correspond in this case to more than one classification. J-pruning now compares the $\mathrm{J}$-values of rule (1) and rule (3). If the J-value of rule (3) is higher than the J-value of rule (1) then node ' $\mathrm{D}$ ' is expanded to rule (2) and (3). If the J-value of rule (3) is smaller than the J-value of rule (1) then the branch that extends rule (1) to rule (3) is truncated and rule (1) completed by assigning it to the majority class of the instances attached to it.

However the calculation of the J-value assumes that we know the right hand side of the rule as well which is in case of classification rules the actual classification. In the case of rule (1) Bramer proposes to assume that the rule predicts the majority class of the instances attached to node ' $D$ '. In the case of rule (3) it is not obvious as the rule may be extended by further branches and thus may change. However Bramer proposes to use in this case the majority class of the instances covered by rule (3).

Similar to J-pruning for Prism algorithms, J-pruning for TDIDT does not exploit the full potential of the $\mathrm{J}$-measure as again it is possible that the J-measure for rule (3) goes up after expanding further branches. Hence applying Jmax-pruning rather than J-pruning may increase the classification accuracy. This could be done by expanding the subtree fully by labelling each intermediate node with the actual J-value based on the majority classification of the training instances attached to the node. After the subtree is induced the node with the highest J-value is identified and the branches are cut down to this node which is then labelled with the majority class. 
Also Jmax could be used here to identify if the J-value is likely to improve at all, as is described for Jmax-pruning in Section 3.2.

A open question remains with $J$-pruning in TDIDT. Using the example from figure 1 and assuming that the J-value of rule (1) is larger than the J-value of rule (3) and that the majority class of the instances at node (1) is ' 1 '. Then rule (3) would be truncated and the two finished rules at this subtree are now:

$$
\text { (1) IF }(A=0) \text { AND }(B=0) \text { AND }(C=0) \text { THEN class }=1
$$

and

$$
\text { (2) IF (A=0) AND (B=0) AND (C=0) AND (D=0) THEN class }=1
$$

Both rules are illustrated in figure 2. We call this kind of rule redundant, as one rule is incorporated totally by the truncated rule including its classification.

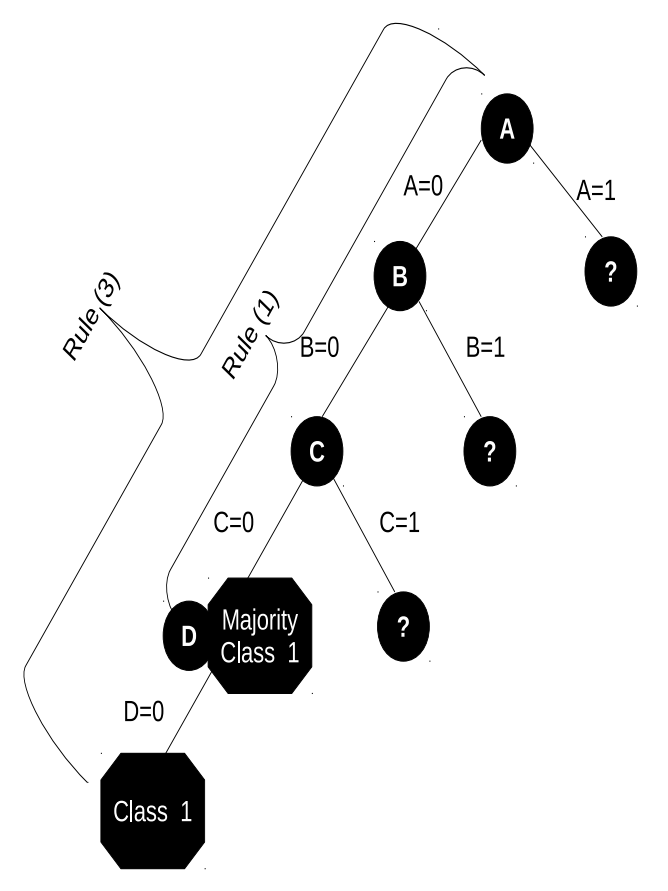

Fig. 2 Example of a decision tree with a redundant rule

As can be seen here rule (1) is completely incorporated in rule (2) and assigned to the same classification, hence also rule (2) can safely be truncated. However assuming that the majority class at rule (1) is ' 2 ', then a different picture emerges. Both rules do not fit into a decision tree and this situation may also arise using 
Jmax-pruning. Thus in the future work we will also investigate how to handle classification rules of which one rule is incorporated into the other rule except for their classifications.

\section{Conclusions}

Sections 1 and 2 discussed an important alternative approach to classification rule induction to TDIDT. The Prism family of algorithms was highlighted and J-pruning, a pre-pruning facility for Prism algorithms based on the J-measure, which describes the information content of a rule, was introduced. Section 3 criticised $J$-pruning as it does not fully exploit the potential of the J-measure. The J-value of a rule may go up or down when rule terms are appended to the rule. J-pruning truncates a rule as soon as the J-value decreases even if it may recover (increase again). The proposed Jmax-pruning exploits the possibility of a J-value recovery and achieves in some cases, examined in Section 4, better results compared with J-pruning, but in every case examined Jmax-pruning achieved at least the same or a higher classification accuracy compared with J-pruning.

The ongoing work comprises the development of J-PrismTCS, a version of PrismTCS that is solely based on the J-measure, by using it also as a rule term selection metric as discussed in Section 5.1. Furthermore the ongoing work comprises the development of a TDIDT algorithm that incorporates Jmax-pruning as discussed in Section 5.2.

\section{References}

1. C L Blake and C J Merz. UCI repository of machine learning databases. Technical report, University of California, Irvine, Department of Information and Computer Sciences, 1998.

2. M A Bramer. Automatic induction of classification rules from examples using N-Prism. In Research and Development in Intelligent Systems XVI, pages 99-121, Cambridge, 2000. Springer-Verlag.

3. M A Bramer. An information-theoretic approach to the pre-pruning of classification rules. In B Neumann M Musen and R Studer, editors, Intelligent Information Processing, pages 201212. Kluwer, 2002.

4. M A Bramer. Inducer: a public domain workbench for data mining. International Journal of Systems Science, 36(14):909-919, 2005.

5. J. Cendrowska. PRISM: an algorithm for inducing modular rules. International Journal of Man-Machine Studies, 27(4):349-370, 1987.

6. F Esposito, D Malerba, and G Semeraro. A comparative analysis of methods for pruning decision trees. IEEE Transactions on Pattern Analysis and Machine Intelligence, 19(5):476491, 1997.

7. E B Hunt, P J Stone, and J Marin. Experiments in induction. Academic Press, New York, 1966.

8. R Kerber. Chimerge: Discretization of numeric attributes. In AAAI, pages 123-128, 1992. 
9. R S Michalski. On the Quasi-Minimal solution of the general covering problem. In Proceedings of the Fifth International Symposium on Information Processing, pages 125-128, Bled, Yugoslavia, 1969.

10. R J Quinlan. C4.5: programs for machine learning. Morgan Kaufmann, 1993.

11. P. Smyth and R M Goodman. An information theoretic approach to rule induction from databases. Transactions on Knowledge and Data Engineering, 4(4):301-316, 1992.

12. F T Stahl. Parallel Rule Induction. PhD thesis, University of Portsmouth, 2009.

13. F T Stahl, M A Bramer, and M Adda. PMCRI: A parallel modular classification rule induction framework. In MLDM, pages 148-162. Springer, 2009.

14. I H Witten and F Eibe. Data Mining: Practical Machine Learning Tools and Techniques with Java Implementations. Morgan Kaufmann, 1999. 